\title{
SHELL PROFILES IN Be STAR SPECTRA
}

\author{
R. W. HANUSCHIK* \\ Astronomical Institute, Ruhr-Universität, D-44780 Bochum, Germany
}

\section{Problem}

Although very different in shape, Be-type and Be-shell-type spectral lines are thought to arise from the same kind of circumstellar environment, a disklike envelope. The only discrimination between these two types of spectra is the inclination angle: If sufficiently high, a large column depth of circumstellar material causes partial obscuration of the stellar disk, i.e. shell absorption; otherwise a pure emission line is observed. A shell line can be considered as equivalent to $P$ Cygni-type absorption trough for different geometry (disk instead of sphere) and kinematics (rotation instead of outflow; see Hanuschik, these proceedings).

Obviously, this very pronounced difference in line shape can be used to determine the average opening angle of a Be star disk. This is, however, difficult in practice since there seems to be nothing like a unique definition of "shell profile" in the literature. The mostly used criterion - central depression (cd) in Balmer lines visible below (or even above) stellar continuum - depends on spectral resolution and is ambiguous because of other effects influencing this parameter like underlying stellar absorption profile, additional circumstellar emission, kinematical broadening. Clearly, a reliable and objective definition of "shell line" is desirable.

\section{Fe II line profiles}

Circumstellar $\mathrm{Fe}$ II lines are the key to the problem because their shape is not distorted by most of the above effects. The straightforward shell criterion for these lines is $I_{\text {cd }}(\mathrm{FeII})<I_{*}$. However, they are not always visible in Be star spectra, while $\mathrm{H} \alpha$ is (per definition). I have therefore searched for spectra with both $\mathrm{H} \alpha$ and $\mathrm{Fe}$ II lines, derived an $\mathrm{H} \alpha$ shell criterion from the above Fe II criterion, and applied this to a large sample of Be and shell-type stars. The $\mathrm{H} \alpha$ parameter is $I_{\mathrm{p}} / I_{\mathrm{cd}}\left(I_{\mathrm{p}}=\right.$ mean double peak intensity). Figure 1 shows the relation of these two parameters. Clearly, the domain with

$$
I_{\mathrm{p}} / I_{\mathrm{cd}}(H \alpha) \geq 1.5
$$

* Now at: Astronomical Institute, University of Tübingen, D-72076 Tübingen, Germany 


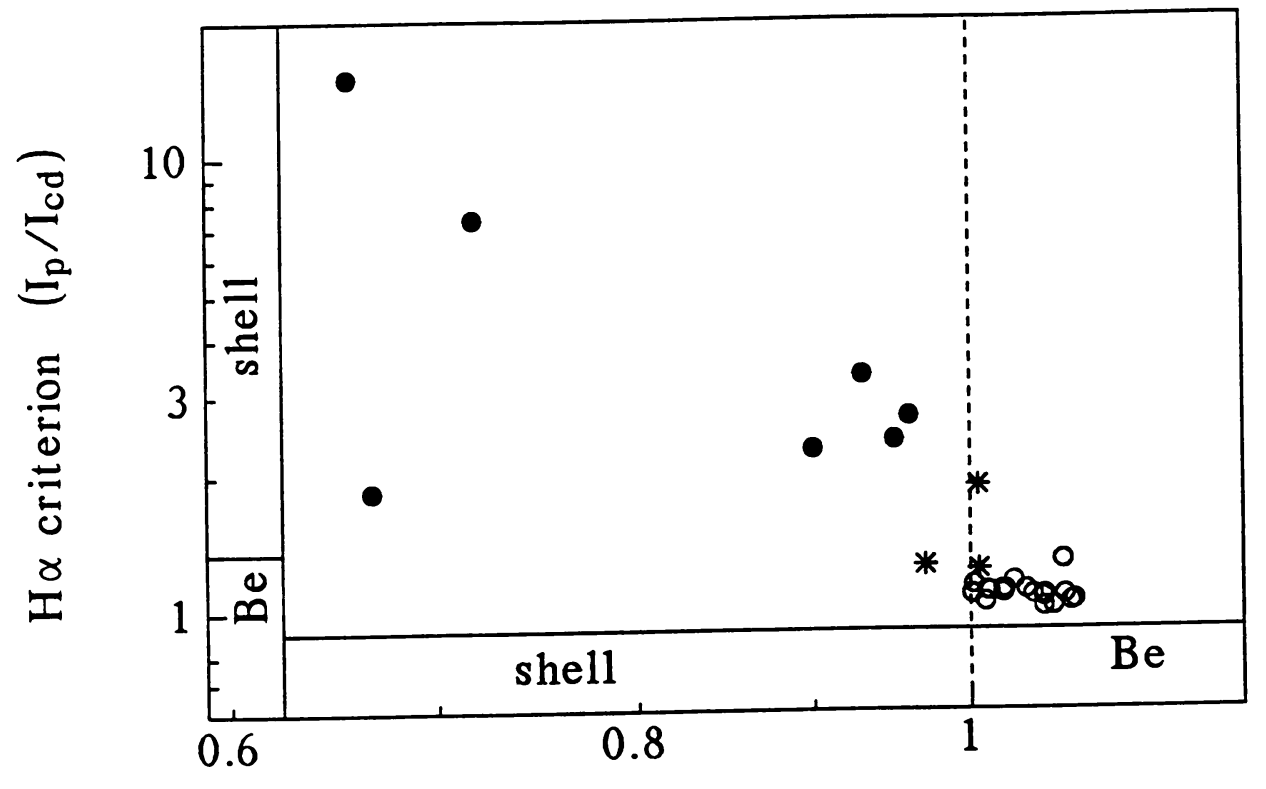

Fe II criterion $\left(\mathrm{I}_{\mathrm{cd}} / \mathrm{I}_{x}\right)$

Fig. 1. H $\alpha$ criterion $I_{\mathrm{p}} / I_{\mathrm{cd}}$ vs. Fe II criterion $I_{\mathrm{cd}} / I_{*}$ for a sample of programme stars with both $\mathrm{H} \alpha$ and $\mathrm{Fe}$ II $\lambda 5317$ measurements available.

is exclusively occupied by shell stars, giving confidence to adopt this criterion in the following.

Applying it to a sample of 109 stars from our atlas (Hanuschik et al., 1994) and that of Doazan et al. (1991), I find that $p=21 \%$ of the spectra are shell spectra. Assuming random distribution of inclination angles $i$, the average half opening angle $\alpha=90^{\circ}-\arccos p$ comes out as $12^{\circ}$. This value is in approximate agreement with results from model line profile calculations assuming a Keplerian disk in hydrostatic equilibrium (Hummel 1993).

\section{Acknowledgements}

I would like to thank Wolfgang Hummel for many valuable discussions.

\section{References}

Doazan V., Sedmak G., Barylak M., Rusconi L.: 1991, ESA SP-1147.

Hanuschik R.W. et al.: 1994, in preparation.

Hummel W.: Ph. D. thesis (Bochum). 